\title{
The Cosmic Infrared Background Experiment (CIBER): Instrumentation and First Results
}

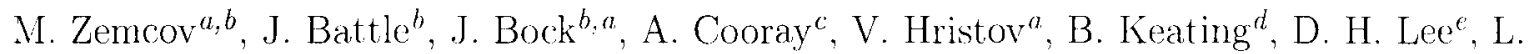 \\ Levenson $^{a}$, P. Mason ${ }^{a}$, T. Matsumotof ${ }^{f, g}$, S. Matsuura ${ }^{f}$, U. W. Nam ${ }^{e}$, T. Renbarger ${ }^{d}$, I. \\ Sullivan $^{a}$, K. Suzuki ${ }^{h}$, K. Tsumura ${ }^{f}$, and T. Warka ${ }^{\prime}$ \\ ${ }^{a}$ Department of Physics, Mathematics and Astronomy, California Institute of Technology, \\ Pasadena, CA 91125. USA; \\ ${ }^{b} J$ et Propulsion Laboratory (JPL), National Aeronauties and Space Administration (NASA), \\ Pasadena, CA 91109, USA; \\ cCenter for Cosmology, University of California, Irvine, Irvine, CA 92697, USA; \\ ${ }^{d}$ Department of Physics, University of California, San Diego, San Diego, CA 92093, USA; \\ ${ }^{e}$ Korea Astronomy and Space Science Institute (KASI), Daejeon 305-348, Korea;

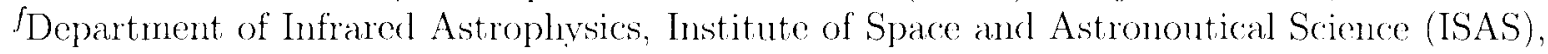 \\ Japan Aerospace Exploration Agency (JAXA), Sagannihara, Kanagawa 252-5210, Japan; \\ ${ }^{9}$ Department of Physics and Astronomy, Sooul National University, Sooul 151-712, Korea; \\ hinstrument Development Group of Technical Center, Nagoya University, Nagoya, Aichi \\ 464-8602, Japan
}

\begin{abstract}
Ultraviolet emission from the first generation of stars in the Universe ionized the intergalactic medimm in a process which was completed by $z \sim 6$ : the wavelength of these photons has been redshlifted by $(1+z)$ into the near infrared today and can be measured using instruments situated above the Earth's atmosphere. First flying in February 2009, the Cosnic Infrared Background ExpeRinent (CIBER) comprises four instruments housed in a single reusable somnding rocket borne payload. CIBER will measure spatial anisotropies in the extragalactic IR background cansed by cosmological structure from the epoch of reionization using two broadband inaging instruments, make a detailed characterization of the spectral shape of the IR backgromed using a low resolution spectrometer, and measure the absolute brightness of the Zodiacal light foreground with a high resolution spectrometer in each of our six science fields. The scientifie motivation for CIBER and details of its first and second flight instrumentation will be discussed. First Hight results on the color of the zodiacal light aromod $1 \mu \mathrm{m}$ and plans for the future will also be presented.
\end{abstract}

Keywords: astronomy, infrared, backgrounds, anisotropy, imaging, spectroneters, telescopes, space optics

\section{INTRODUCTION}

The extragalactic background light (EBL) is the sum of all of the light enitted along a line of sight through the Universe. At near infrared (IR) wavelengthes, the EBL is predominantly due to stellar light: a measurement of the EBL at these wavelengths is a census of the total energy released by the process of nucleosynthesis through the history of the Universe (see [1] for a comprehensive review). However, to date absolute measurenents of the EBL at near IR wavelengths lack the accuracy to constrain the radiative content of the cosmos to within an order of magnitude ([2], [3], [1], [5], [6], [7], [8]). Further, the contribution to the EBL from galaxies does not reproduce the near IR EBL neasured by instruments which perform absolute photonnetry of the background light. For example, at $\lambda=3.6 \mu \mathrm{m}$ the EBL measured by DIRBE using absolute photometry is $12.4 \pm 3.2 n \mathrm{~W}$ $\mathrm{n}^{-2} \mathrm{sr}^{-1}$ [9], while the decpest pencil bean surveys with Spitzer yield $6-9 \mathrm{nW} \mathrm{m} \mathrm{m}^{-2} \mathrm{sr}^{-1}$ ([10], [11]). At shorter wavelengths this discrepancy is even more pronounced: this leaves open the possibility that there exists some

E-mail: zemcov@caltech.edu

Ground-based and Airborne Instrumentation for Astronomy III, edited by lan S. McLean

Suzanne K. Ramsay, Hideki Takami, Proc. of SPIE Vol. $7735,77351 \mathrm{~W} \cdot($ C) 2010 SPIE C CC code: $0277-786 \times / 10 / \$ 18 \cdot$ doi: $10.1117 / 12.857483$

Proc. of SPIE Vol. $773577351 \mathrm{~W}-1$

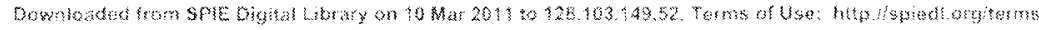


truly diffuse emission comprising a fraction of the near IR background and that using suitable instruments it. may be possible to measure it.

Importantly, as the EBL traces star formation throughout the history of the Universe, it contains information about the earliest generation of stars which were responsible for ionizing it (see [12] for a review). As the early Universe contained only an electrically neutral gas composed predominantly of hydrogen, it was opaque to rest frame photons shorter than the Lyman- $\alpha$ transition at $122 \mathrm{rm}$. Such ultraviolet (UV) photons were efticiently absorbed by electrons occupying the ground state of hydrogen so that such photons had a very small mean free path, which is to say, photons were absorbed so quickly that neighlouring regions were invisible to one another. This cosmological "dark age" ended when the first generation of stars and their associated stellar products and remnants ionized the neutral medimn in the early Universe in a relatively brief epoch which present measurement." show was underway by a redshift $z \approx 10$ and almost complete by $z \approx 5$. This period is known as the epoch of reionization. Since cosmological redshifting lengthens the wavelength of photons, the UV photons emitted by these stars are now observable in the near IR. While the spectrum of the near IR EBL does contain information about the epoch of reionization, it is expected to be fant compared to the contribution from galaxies. However. as the signature from the first stars is expected to be both diffuse and structured on large scales, the spatial fluctuations of its imprint on the near IR EBS yield a great deal of information about the formation of structure during the time of the first stars [13].

The cosmological dark ages were a critical tine for the formation of structure in the Universe during which matter collapsed under gravity into over dense regions secded in the early Universe to form the first stars and galaxies. Wufortunately, it is also the most difficult to study as what little electromagnetic radiation was being produced by the mater during this process was efficiently reabsorbed by the ubiguitous neutral medium. This means that the signals from this epoch are faint and that the matter in the Universe which has been radiating since that reionization generates a strong foreground to the measurement. Instrunents which can measure quantities from this crucial time in the history of the cosmos are therefore both scientifically important and technically difficult.

The dominant source of photens in the near IR band near the Earth is the Zodiacal light (ZL): this source of diffuse sky brightuess is due to sunlight scattering off small chust particles lying in the plane of the oclipt ic Barring leaving the plane of the ecliptic or the solar system entirely, it is a foregromel to the measurement of the EBL which must be managed carefully. Additionally, there are sources of diffuse emission and scantering associated with the galaxy which, though significantly fainter than the ZL, must also be understood before the EBSL can be reliably measured.

The Cosmic Infrared Background ExpeRiment (CIBER) is a program of someling-rocket borne packages of instruments designed to measume both the spectrum of the EBL and the spatial fluctuations in the EBL imprinted during the epoch of reionization. The first CIBER payload will probe the power spectrum of near IR FBI uctuations for a first light galaxy-EIBL component, limit the strength of the Lyman cute signature of reionization in the near IR EBS between the optical and near-infrared EBL measurements, and measure the EBL from $0.7-2.1 \mu \mathrm{m}$ down to the instrumental zodiacal foreground subtraction linit. In this paper the design and implenentation of the curent. CIBER hardware are discussed, and science results from its first flight are presented.

\section{THE CIBER PAYLOAD}

The CIBER payload comprises two inaging telescopes ("Inagers") which are designed to study the spatial fluctuations of the diffuse near IR backgromed, a low resolution spectroneter (LRS) designed to measure the spectrum of the EBL in the range $0.7 \leq \lambda \leq 2.1 /$ m, and a narrow band spectrometer (NBS) designed to characterize the absolute brightness of the ZL in the CIBER fields using the CaII Framhofer line[14]. Below we present the design of the payload and electronics systems and discuss the interfaces to the sounding rocket itself.

\subsection{Optics and Cryogenic Components}

A model of the CIBER cryogenic insert is shown in Figure 1. The sounding rocket defines the coordinate system of the payload, with rocket aft (this is, towards the motors) at the instrument aperture side, and rocket

Proc. of SPIE Vol. $773577351 \mathrm{~W}-2$ 
forward (towards the nose) in the direction towards the experiment's cryostat. Due to its low density and strength, Aluminum 6061 is used throughout the CIBER instrument assembly except where noted. Starting at the forward end, the experiment section comprises a $7 \ell$ liquid nitrogen vessel filled with an open-cell aluminmm foam; the foam ensures that under zero- $y$ conditions the liquid stays in thermal contact with the metal due to its large surface area. Just forward of the cryostat's work surface are attached 12 G-10 plates which mate to an almminum pressure bulkhead. This bulkhead interfaces to the rocket sections forward of the experiment (see Section 2.3) and has hermetic connections through which the electronic cabling runs.

\section{$\mathrm{LN}_{2}$ Cryostat}

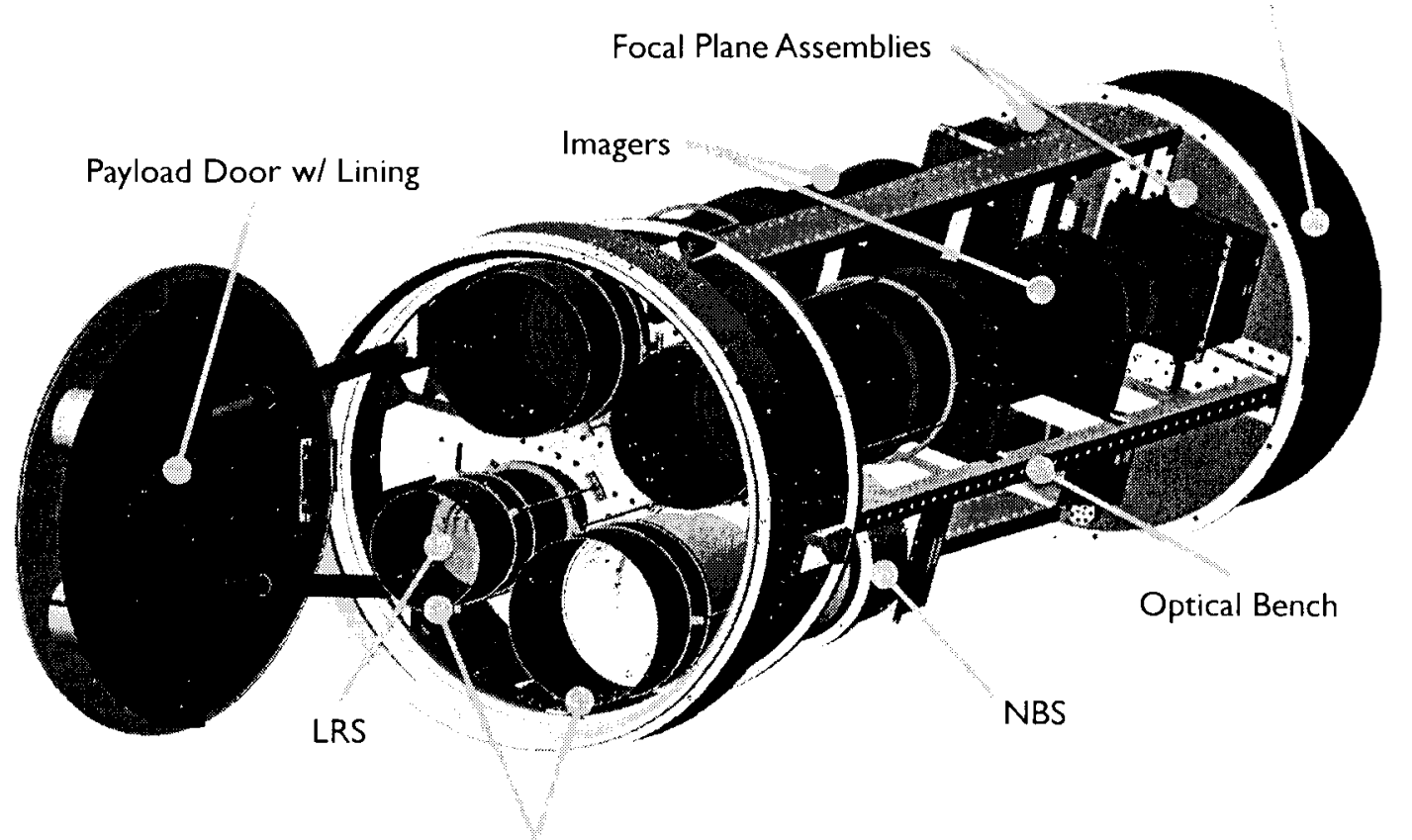

Pop Up Baffles

Figure 1. The CIBER cryogenic insert - see the text for a detailed description. This section (exchuding the door) is housed in an aluminum cylinder which, when mounted at the fore and aft by the pressure bulkhead and door, is vacuun tight. This cylinder also serves double-duty as the skin of the rocket.

Mounted to the cryostat's aft end is the optical bench to which the instruments are attached. Figure 1 shows the configuration of the instruments; all four assemblies were designed and manufactured by the Genesia Corporation of Japan using specifications developed by the science team. The optical bench is machined to a thickness of $19 \mathrm{~mm}$ and is light weighted along its length. Structural spines are mounted to the top and bottom of the optical bench to increase the strength of the full assembly. Due to the themal loading from the instruments on the optical bench, the temperature of its forward side is typically $78 \mathrm{~K}$ while the temperature of the aft side is typically $85-90 \mathrm{~K}$. Attached to the aft end of the bench is a plate which stiffens the assembly, interfaces the optical bench to the front end Titanium suspension. and provides a stable base for the pop up bafle assemblies. The front plate has holes through which the four instrument's static baffles protrude. The whole cryogenie insert is surrounded by cylindrical radiation shields; these are fabricated from Aluminum 1100, reinforced with 
Table 1. CIBER Instrument Specifications

\begin{tabular}{lccc}
\hline & Imagers $^{\circ}$ & LRS & NBS \\
\hline Aperture & $11 \mathrm{~cm}$ & $5 \mathrm{~cm}$ & $7.5 \mathrm{~cm}$ \\
Pixel size & $7^{\prime \prime} \times 7^{\prime \prime}$ & $1.4^{\prime} \times 1.4^{\prime}$ & $2^{\prime} \times 2^{\prime}$ \\
FOV & $2^{\circ} \times 2^{\circ}$ & 5 slits, each $5.5^{\circ} \times 2.8^{\prime}$ & $8^{\circ} \times 8^{\circ}$ \\
$\lambda$ & $1.2 / 1.6^{\circ} \mu \mathrm{m}$ & $0.7-2.1 \mu \mathrm{m}$ & $854.2 \mathrm{~mm}$ \\
$\delta \lambda / \lambda$ & 0.5 & & \\
$R$ & & $15-25$ & 1200 \\
Optics QE & 0.85 & 0.8 & 0.65 \\
\hline Array QE & 0.7 & 0.8 & 0.8 \\
Photo current $\left(\mathrm{e}^{-} / \mathrm{s}\right)$ & 15 & 0.6 & 0.5 \\
Dark current $\left(\mathrm{e}^{-} / \mathrm{s}\right)$ & 0.3 & $<0.1$ & $<0.1$ \\
Read noise $\left(\mathrm{e}^{-}\right)$ & 15 & 26 & 28 \\
\hline
\end{tabular}

${ }^{a}$ CIBER has two imagers on board, nominally centered at astronomical I-band and $\mathrm{H}$-band.

Aluninum 6061 ribs, hard black anodized on their interior surface. mechanically supported at the front plate and thermally and mechanically tied to the cryostat directly at the forward end of the insert. These shields ensure a low radiative load on the optical bench and instruments over most of their length.

The optical bench spines also host the (anlibration lanp assemblies. Each assembly consists of an IED) (onpled to a optical fiber, which is routed to an instrument. These fibers are coupled to the instrument's optics by means of a small finger with a 45 degree mirror mounted at its exit aperture; light emitted by the LFD is fed through this assembly and scatters off the instrunent sopties. which illuminates the dedector assembly in a non-muifom but repeatable way. The LFI peak wavelength, driver current and geonetric attenution in the calibration lamp) systen is tuned for each instrument to yield a photo current $\sim 100 e^{-} / \mathrm{s}$ in each instrument.

The specifications for the instruments themselves are described clsewhere ([14], [15]); we summarize them in Table 1 for reforence. To hold the detector arrays at their optical focus, all four instrumentis employ a mechanical focal plane assembly, a model of which is shown in Figure 2. The detector arrays are held in commercially available chip carriers which are interfaced to the electronics via a focal plane board (FPB, see Section 2.2). The FPB is mechanically monnted into a light tight box. This box has a frame against which the detector chip is pressed by a spring loaded plunger assembly; the frame and plunger assembly define the plane on which the detector array rests. The spring tension is carefully chosen so as not to stress the detector array itself.

The FPB light box is mechanically monuted to the instrument interface plate by an assembly designed to allow active thermal control of the detector array and focal plane board. The Fl'B light box interfaces to an Aluminum 1100 structure which contains a themal choke. A themal control unit is placed at this choke point: this unit contains a hoater element and platinmm-thermometer bridge circuit that allows active control of the temperature of the choke point. The thermal control unit is attached to the instrument interface plate via another Alumimm 1100 structure and four steel legs which serve to increase the thermal time constant between the FPB light box assembly and the ambient thernal enviromment. An outer light tight box is then nomnted to the instrument interface plate over the FPB light box and thermal control assembly: this ensures that stray photons and radiative loads cannot couple to the imer light tight box and detector arrays. This focal plane assembly (FPA) is attached to the instrunent; themal isolation of the Fl'A from the instrunent body is achieved throngh the use of long steel screws threaded through Vespel' posts. For each instrument, the FPA is attached to the cryostat directly via high thermal conductance copper braids.

Each FPA also hosts a cold shutter assembly based on a flight-proven design [16]. These shutter's are composed of thin, hard black anodized aluninum blades mounted on a flexural pivot and counter weighted with small rareearth magnets. The shutters are actuated by an electronagnet at ached to the muderside of the shutter mount: the magnetic flux is routed to the level of the shutter blade using a small $\mu$-metal spur. The shutters flip state when current is applied to the electromagnet, and are extremely stable when no current is applied.

\footnotetext{
${ }^{1}$ See http://www2.dupont . com/Vespel/en_US/products/s/sp3.html.
} 


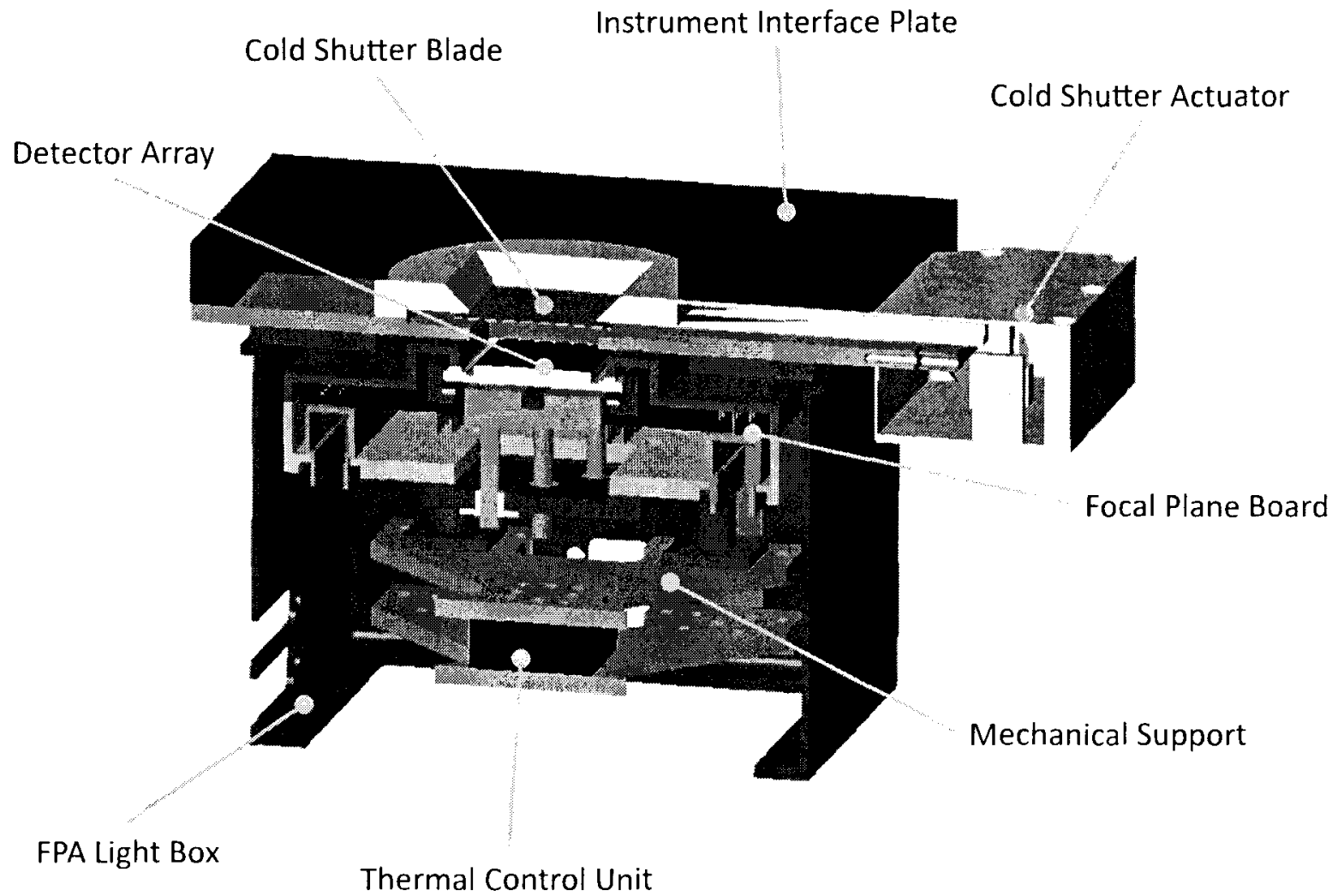

Figure 2. A model of a single CHBFR's focal plane assembly (FPA); each of the four instruments uses an FPA to hold the detector array at the focus of its optics. $A$ detaled discussion of the FPA is given in the text.

CIBER's first flight showed that, in its original configurntion, there was a significant problem with scattered themal radiation increasing the photo current present in the instruments when observing the sky. It was later determined that the dominant scattering path is from the part of the skin and door assembly beyond the instrument's static baffles. On ascent, the rocket skin is flash healed by atmospheric drag to temperatures above $400 \mathrm{~K}$. Though the scattering compling between the instruments and the hot skin is small, the absolute brightness of the thernal radiation is large; this leads to an instrunent-dependent but large scattered signal at the detectors. To combat this problem, a number of changes have been instituted for CIBER's second flight. Most importantly, pop up baffles have been installed at the front plate of the experiment section on all foum instruments (see Figure 1). These baffles are slightly larger than their static counterparts and are designed to extend when the experiment door is open to just beyond the lip of the rocket skin. The baffles themselves are made of $A$ luminum 6061 with either hard black anodization (for instruments with passbands sensitive to $\lambda \lesssim 1.6 \mu \mathrm{m}$ ) or Fpner laser black ${ }^{2}$ (for instruments sensitive to $\lambda \geq 1.6 \mu \mathrm{m}$ ) on their interior surface. These baffles ride on steel rods run through brass bushings and are spring loarded so they extend when the door is opened. Measurements of the improvement in the instrunents' semsitivity to seattered off-axis light have shown that these reduce the expected thermal pick up by as much as a factor of $10^{3}$ for the geometries expected in flight. In addition to the pop up baffles, additional radiation shields have been added to the front section of the payload extending beyond the front plate, and to the (warm) surface of the door. When the experiment is

${ }^{2}$ See http://www.epner.com/laser_black.ssi. 
cooled, the pop up baffles and door shields radiatively cool to $\sim 150 \mathrm{~K}$ and $\sim 210 \mathrm{~K}$, respectively, while the front radiation shield extension cools to $\sim 90 \mathrm{~K}$ via the optical bench. Together, these changes should mitigate the effects of thermal scattering on the instrument's performance.

\subsection{Detectors and Electronics}

CIBER's electronic chain achieves three main objectives:

1. Bias and read out the detector arrays.

2. Digitize the analog detector ontputs, incorporate housekeeping data, and pass them to the rocket telemetry system.

3. Receive the telemetered data and store it to disk.

The first and second tasks are accomplished through the combination of the FPB discussed in Section 2.1 and a warm array processor board $(\Lambda P B)$. The $\Lambda P B$ accepts clocking signals generated by master clocks on the rocket telemetry system and generates the necessary clock signals required by the detector array's multiplexer. 'Theste clocks are sent to the the FPB through a custom wiring harness which comprises $300 \mathrm{~K}^{-3}$ and cryogenict sections interfaced through commercial hermetic commections; this harness is shared by the return analog detector output signals.

In total, CIBER utilizes fom commercially availables detector arrays; two $1024 \times 1024$ pixel IIAWAII-1 architecture detector arrays for the inagers and two $256 \times 256$ pixel substrate renoved PICNIC architecture detector arrays for the spectrometers. These arrays are helel at $\sim 81 \mathrm{~K}$ when operating; their temperature is actively controlled using the thermal choke discussed in section 2.1. Both types of array are biased and read out using the scheme presented in [17]; the FPB simply implements their bias and readout strategy. The' major differenee betwen the CIBER and the $|17|$ readont selemes is the use of a dual (as opposed to single-sirled) follower circuit at the array rearlont. The currents from the array are converted to voltages using a matched dual JFET follower: the matching ensures that common mode noise and interference experienced by the array readout is tracked by a reference voltage and can be differenced out of the array output.

Both sides of the JFF' output, are brought ont to the APB and then differenced, and an amplification factor of $10 \times$ is applied to the resulting differential voliage. This analog signal is then digitized using a 16 bit anatog to digital converter chip. Becanse the serial clock speed is $10 \mathrm{Mbs}$ and an detector pixel's signal is digitized using 16 bits, the array pixels are clocked at a rate of $1.6 \mu \mathrm{s} /$ pixel; this leads to a frame rate of $1.78 \mathrm{~s}$ for each HAWAll-1 array and $0.26 \mathrm{~s}$ for each PICNIC arrays. The resulting digitized output is synchronously sent from the $\mathrm{AP} B$ to the rocket telenetry system for incorporation into the rocket data strean and real tine transmission to the gromud stations (see Section 2.3).

The raw rocket telemetry is received and processed by the gromul station and sent onward to the experiment ground station electronies (CSE) using a NASA system [18]. To implement the real time image display and archiving both when integrated with the rocket and under test, the CIBER GSE system has following three modes:

1. Serial mode simulates the rocket telemetry for the CIBER instrument providing power, serial clocks and command signals.

2. Parallel mode receives and parses the experiment data via three rocket telemetry chanmels (this mode is used in flight).

3. Common mode displays and archives all the instrument data for both serial and parallel modes.

\footnotetext{
${ }^{3}$ http://www. stonewallcable.com/

${ }^{4}$ http://ww. tekdata-interconnect.com/

${ }^{5}$ These arrays are manufactured by Teledyne Scientific \& Imaging LLC: http://www.rockwellscientific.com/imaging/standard_products.html
} 
The CIBER GSE uses a data acquisition card ${ }^{6}$ (DAQ) for its high-speed interfaces. A DSP hoard handles commands from the graphical user interface software coded for the CIBER, and transfers data from the interface boards to the DAQ card in the PC: custom-written software is then used to store these data to disk and for real time visualization. The serial interface boards, connected directly to the instruments, are used in the serial mode and the parallel interface boards, comected to the experiment via the telemetry and NASA ground station, are used in the parallel mode.

\subsection{Rocket Payload}

And overview of the NASA Sonnding Rocket Program can be found in e.g. [18] and [19]; the CIBER experiment section is designed to launch on the Black Brant family of vehicles, the largest in the NASA stable. From aft to forward, the CIBER payload stack consists of the ignitor housing for the motors, the high velocity spring separation section, the experiment section itself, the startracker housing, the telemetry section, the active guidance section, the celestial attitude control system, and the ogive recovery system assembly. The CIBER section mechanically interfaces to the rocket motor and ignitor system at the aft side via the experiment door section, and at the fore sicle by a radial-axial ("radax") joint. The payload is a miform 17.26 " in clianeter along its length. Together, these systems provide launch and separation capability active pointing while in flight. real time telenetry, and parachute recovery. These systens are all standardized with interchangeable parts except the telemetry and startracker sections which are customized for the experiment.

The CIBER flight sequence is typical for this class of rocket. At launch, the rocket burns its Mark 70 Terrier first stage; the burn lasts for approxinately $6 \mathrm{~s}$ and then is drag separated from the second stage. Guide fins at the base of the motor are canted by a small angle to give the rocket an $\sim 4$ Hz roll rate; this stabilizes the rocket by giving it an angular momentum vector pointing along its length. The second stage motor, a Black Brant mod 2, burns for approximately 30 s and is pushed away from the payload by the high velocity spring section attached to the top of the motor. To shed some of the angular momentum recpured during the burn, two cables with weights at their ends are released from the payload to despin it. When the cables reach their full extent they are released; following this, the payload is spinning at $\sim 0.5 \mathrm{~Hz}$. The experinent shut ter door then opens and observations are performed (Section 3). At the end of the Hight before re-entry, the door is closed and gas is used to spin the payload back up to $\sim 1 \mathrm{JIz}$. The parachute deploys at an altitude of 16 , $000 \mathrm{ft}$ and the payload impacts at about $30 \mathrm{ft} / \mathrm{s}$.

The CIBFR payload's telemetry section uses $3 \times 10 \mathrm{Mbs}$ downlinks, one for each inager and one for the? combination of both spectrometers and rocket housekeeping data. These links are tramsmitted over wrap-around antemas fitted in recesses on the rocket skin transmitting in three separate bands between 2215 and $2280 \mathrm{MH}$. The startracker section uses a University of Wisconsin developed model ST-5000 sidelooking star tracker [20)]. The CIBER payload is the first deployment of such a system, whose advantage is that it need not be co-mounted at the instrunent aperture (and thus take space from the science instrunents). The side looking star tracker is aligned with the instruments using a secondary, temporarily installed ST-5000 system which is co-nomited to the a.ft bulkhead. This temporary tracker is optically aligned to the instrmuents; the offscts to the side looking tracker are deternined by using the celestial pointing system to measure stars' positions at night in both trackers and solving for the Euler rotations between them.

\section{CIBER'S FIRST FLIGHT}

A Terrier-Black Brant IX rocket carrying the CIBER payload was successfully launched from White Sancts Missile Range on 25 February, 2009. It achieved an apogee of $\sim 330 \mathrm{~km}$ and provided 425 seconds of astronomical data. The calibration lamps and cold shntter's were operated during the ascent and descent phase to monitor the performance of the detectors maler flight conditions. During the flight the payload's pointing stability was $<3^{\prime \prime}$ in a given field, with $<10^{\prime}$ absolute pointing error. Following landing in the desert, the instrunent was successfully recovered for future flights.

The choice of our science fields is discussed in detail in [15]. Since the Boötes, North Ecliptic Pole and SWIRE ELIAS-N1 fields are well studied with many other near IR telescopes, these fields were chosen for the CIBER

\footnotetext{
${ }^{6}$ Model number DAQ6534 from National Instruments.
} 
imaging instruments. The Elat-10 ("Echiptic Latitude 10 degrees") and Elat-30 fields were chosen to measure the zodiacal light intensity as a function of ecliptic latitude. The remaining science fields have higher ecliptic latitudes to reduce the zodiacal foreground's brightness for the extragalactic light measurements. These EBL fields can be used to constrain the ZL as well, since the ZL is always brighter than the extragalactic signal.

[15] gives results from the first flight; in summary, CIBER measured a very red ZI spectrum in the wavelength range $0.7<\lambda<1.4 \mu \mathrm{m}$ which is qualitatively consistent with the hypothesis that $\mathrm{ZL}$ dust originates from S-type asteroids. In addition, the spectrum of the star 42 Draconis was neasured over the sane wavelength range at the highest spectral resolution to date. Interestingly, the star's spectrum shows features not reproduced by stellar models. Finally, the NBS measured the flux from the star Vega at 854 mm to better than $1 \%$ accuracy.

CIBER is scheduled to launch again in .July 2010 on another Black Brant IX with a similar flight profile to the first launch. A number of changes to the payload hardware, detailed in Section 2.1, have been instituted to reduce the large instrumental backgromeds observed in the first flight. The CIBER program is funded for an additional two flights beyond that, with the fourth flight on a Black Brant XII and no payload recovery. Still beyond that, CIBER-II, the follow on payload to CIBER, has been funded and is expected to begin flying in 2014.

\section{SUMMARY}

From its first flight alone, CIBER has already changed our view of the source of the ZL, and provided new measurements of standard stars for calibration purposes. Using the data acquired during CIBSR's first and future launches additional measurements will also be made; these are smmmarized below.

- Using CIBER's sensitivity, higher angular resolution, and deep point source removal to probe the spectrum of near IR EBL fluctantions for a first light galaxy-EBL component, and combine CIBER data with ancillary surveys for the denitive datal set for EBL uctuations in 4 bands from 0.9 to $3.6 \mu m$.

- A search for the Lyman ent off signature of reionization in the near IR EBL between the optical and near-infrared EBL measurementis will be performed.

- The zodiacal foreground in several fields will be measured using a narrow-band spectrometer to trace scattered near-IR Fraunhofer absorption features.

- The low-resolution and narrow-band spectrometer data will be combined to probe the EBL from $0.7-2.1$ /m down to the narrow-band spectroneters zodiacal foregromel subtraction linit.

\section{Acknowledgments}

This work was supported by NASA APRA research grants (NNX07AI54G, NNG05WC18G, NNX07AG43G, and NNX07AJ24G), and KAKENHI grants (20.34, 18204018, 19540250, 21111004 , and 21340047) from the Japan Society for the Promotion of Science and the Ministry of Education, Culture, Sports, Science and Technology. We acknowledge the dedicated efforts of the somiding rocket staff at N $\Lambda$ S $\Lambda$ Wallops Flight Facility and White Sands Missile Range, and the engineers at the Genesia Corporation for the technical support of the CIBBER optics. We thank Dr. Allan Snith, Dr. Keith Lykke, and Dr. Steven Brown the National Institute of Standards and Technology) for laboratory calibration of the four CIBER instmuments. NZ acknowledges support from a NASA Postdoctoral Fellowship, KT acknowledges support from the JSPS Research Fellowship for the Young Scientists. and $A C$ acknowledges support from an NSF CARRER award.

\section{REFERENCES}

[1] Hauser, M. G. and Dwek, F., "The Cosmic Infrared Backgromnd: Measurements and Implications," ARA $9 A$ 39, 249-307 (2001).

[2] Hauser, M. G., Arendt, R. G., Kelsall, T., Dwek, E., Odegard, N., Weiland, J. L., Frendenreich, H. T., Reach, W. T., Silverberg, R. F., Moseley, S. H., Pei, Y. C., Lubin, P., Mather, J. C., Shafer, R. A., Smoot, G. F., Woiss, R., Wilkinsom, D. T., and Wright, E. L., "The COBE Diffuse Infrared Backgromel Experiment Search for the Cosmic Infrared Background. I. Limits and Detections," $A p J(1998)$.

Proc. of SPIE Vol. $773577351 \mathrm{~W}-8$ 
[3] Dwek, E. and Arendt, R. G., "A Tentative Detection of the Cosmic Infrared Background at $3.5 \mu \mathrm{m}$ from COBE/DIRBE Observations," ApJ (1998).

[4] Gorjian, V., Wright, E. L., and Chary, R. R., "Tentative Detection of the Cosmic Infrared Background at 2.2 and 3.5 Microns Using Gromel-based and Space-based Observations," ApJ (2000).

[5] Wright, E. L., "DIRBE minus 2MASS: Confirming the Cosmic Infrared Background at 2.2 Nicrons." Ap.J (2001).

[6] Cambrésy, I., Reach, W. T., Beichman, C. A., and Jarrett, T. H., "The Cosmic Infrared Background at 1.25 and 2.2 Microns Using DIRBE and 2MASS: A Contribution Not Due to Galaxies?," $A p J(2001)$.

[7] Matsumoto, T., Matsuura, S., Murakami, H., Tanaka, M., Frennd, M., Lin, M., Cohen, M., Kawada, M., and Nola, M., "Infrared Telescope in Space Observations of the Near-Infrared Extragalactic Background Light," ApJ (2005).

[8] Levenson, L. R., Wright, E. L., and Johnson, B. D., "DIRBE Minus 2MASS: Confirming the CIRB in 40 New Regions at 2.2 and $3.5 \mu \mathrm{m}, " A p J(2007)$.

[9] Wright, E. L. and Reese, E. D., "Detection of the Cosmic Infrared Background at 2.2 and 3.5 Microns Using DIRIBE Observations," Ap,I (2000)

[10] Fazio, G. G., Ashby, M. 1. N. Barmby, P., Hora, J. L., Huang, J., Pahre, M. A., Wang, Z., Willner, S. P.. Arendt, R. G., Moseley, S. II., Brodwin, M., Eisenhardt, P., Stern, D., Tollestrup, F. V., and Wright, E. I.., "Number Counts at $3 \mu m i \lambda ; 10 \mu m$ from the Spitzer Space Telescope," ApJS (2004).

[11] Sullivan, I., Cooray, A., Chary, R., Bock, J. J., Brodwin, M.. Brown. M. J. I.. Dey, A.. Dickinson, M.. Eisenhardt, P., Ferguson, H. C., Giavalisco, M., Keating, B., Lange, A., Mobasher, B., Reach, W. T.. Stern, D., and Wright, E. L., "Chustering of the IR Backgromed Light with Spitzer: Contribution from Resolved Sources," Ap.J (2007).

[12] Fan, X., Carilli, C. L.. and Koating, B., "Otservational Constraints on Cosnic Reionization," ARA $(20) 60)$.

[13] Cooray, A., Bock, J. J., Keatin, B., Lange, A. E., and Matsmunoto, T., "First Star Signature in Infrared Background Anisotropies," ApJ (2004).

[14] Bock. J., Battle, J., Cooray, A., Kawada, M., Keating, B., Lange, A., Lee, D., Matsumoto, T., Matsuma. S., Pak, S., Renbarger, T., Sullivan, I., Tsumura, K., Wada, 'T., and Watabe, T., "The cosmic infrared background experiment." New Astronomy Review (2006).

[15] Tsumura, K., Battle, J., Bock, J., Cooray, A., Iristov, V., Keating, B., Leee, D. H., Levensom, L. R., Mason, P., Matsumoto, T', Matsumra, S.. Nam, U. W., Renbarger. T., Sullivan, I., Suzuki, K., Wada, T., and Zemeov, M., "Olsservations of the Near-Infrared Spectrum of the Zodiacal light with CIBER." Ap.l. In Press (arXio/1004.5445) (2010).

[16] Bock, J. J., Kawada, M., Lange, A. E., Matsumoto, T., Uemizu, K., Watabe, T., Yost, S. A.. Fazio, G. G., Forrest, W. J., Pipher, J. L., and Price, S. D., "Rockethorne instrument to search for infrared emission from baryonic lark matter in galactic halos," in [Socicty of Photo-Optical Instrumentation Engineers (SPIE) Conference Series], A. M. Fowler, ed., Society of Photo-Optical Instrumentation Engincers (SIE) Conference Series 3354 (1998).

[17] Hodapp, K., Hora, J. L., Hall, D. N. B., Cowie, L. L., Metzger, M., Irwin, E., Vural, K., Kozlowski. L. J., Cabelli, S. A., Chen, C. Y., Cooper, D. E., Bostrup, G. L., Bailey, R. B., and Kleinhans, W. E., "The HAWAII Infrared Detector Arrays: testing and astronomical characterization of prototype and science-grade devices," New Astronomy (1996).

[18] Krause, D. J., "NASA's Sounding Rocket Program NSROC, accomplishments and the future," in [17th ESA Symposium on European Rocket and Balloon Programmes and Related Research], B. Warmbein, ed., ESA Special Publication $\mathbf{5 9 0}$ (2005).

[19] NASA Sounding Rockets Program Office. The NASA Sounding Rocket Program Handbook.

[20] Percival, J. W., Nordsieck, K. H., and Jaehnig, K. P., "The ST5000: a high-precision star tracker and attitude determination system," in [Society of Photo-Optical Instrumentation Engineers (SPIE) Conference Series], Society of Photo-Optical Instrumentation Enginetrs (SPIE) Conference Series 7010 (2008). 